\title{
Tangence
}

\section{Les bons sentiments}

Amitié et politique dans Two Solitudes de Hugh MacLennan

\section{Jacques Cardinal}

Numéro 63, juin 2000

Fictions et politique

URI : https://id.erudit.org/iderudit/008186ar

DOI : https://doi.org/10.7202/008186ar

Aller au sommaire du numéro

Éditeur(s)

Presses de l'Université du Québec

ISSN

0226-9554 (imprimé)

1710-0305 (numérique)

Découvrir la revue

Citer cet article

Cardinal, J. (2000). Les bons sentiments : amitié et politique dans Two Solitudes de Hugh MacLennan. Tangence, (63), 135-164. https://doi.org/10.7202/008186ar d'utilisation que vous pouvez consulter en ligne.

https://apropos.erudit.org/fr/usagers/politique-dutilisation/ 


\title{
Les bons sentiments. Amitié et politique dans Two Solitudes de Hugh MacLennan
}

\author{
Jacques Cardinal, Université de Montréal
}

\begin{abstract}
Thinking of Heather and Paul, he reflected with wonder and some indignation that each was the victim of the two racial legends within the country. [...] On both sides, French and English, the older generation was trying to freeze the country and make it static. [...] Yet the country was changing. In spite of them all it was drawing together; but in a personal, individual way, and slowly, French and English getting to know each other as individuals in spite of the rival legends (Two Solitudes, p. 301).
\end{abstract}

Voilà déjà un peu plus de cinquante ans qu'a été publié le plus célèbre des romans de Hugh MacLennan, roman dont le titre désigne depuis lors le drame existentiel et politique entre anglophones et francophones au Québec et au Canada ${ }^{1}$. L'intention première du roman est généreuse: tolérance, ouverture, amitié, harmonie entre les sujets sont pour ainsi dire les principes fondateurs d'un discours qui vise à résoudre le conflit qui oppose les deux solitudes depuis longtemps. Pourtant, à relire ce roman audelà de ses intentions, on s'aperçoit qu'il n'est pas sans faire subir à ces principes une certaine (dis)torsion afin que l'on juge autrement de l'histoire politique du Canada. Question d'angle ou d'éclairage qui n'invalide en rien le bien-fondé ou la légitimité de ces principes, mais les fait jouer à l'intérieur d'un discours - et d'une énonciation - dont il convient d'analyser l'incidence.

On se souvient que le roman se veut d'abord, par-delà le conflit politique qui sévit entre le Québec et le Canada, une critique du conservatisme des deux communautés au nom d'un

1. Hugh MacLennan, Two Solitudes, Toronto, Stoddart, coll. "NewPress/Canadian Classics", 1993 (édition originale: MacMillan, 1945). Toutes les citations du roman renvoient à cette édition. Après une citation, j'indique la page entre parenthèses. 
certain humanisme libéral ${ }^{2}$. Certains personnages se présentent en effet comme des citoyens de bonne foi, faisant montre d'objectivité et d'impartialité, ouverts à l'autocritique et aux changements. C'est par ce discours que l'on veut ici résoudre le conflit entre les "two racial legends", en les replaçant justement dans un autre cadre idéologique et politique. Alors qu'on a voulu trop souvent réduire le drame des deux solitudes à une opposition exclusivement ethno-culturelle, le roman cherche plutôt à reconsidérer l'incidence de ce conflit dans le cadre historique de l'évolution des idéologies. De ce point de vue, cette opposition des deux solitudes n'est ni simple ni homogène dans la mesure où elle se complexifie depuis une autre opposition, interne celle-là à chacune des deux communautés. Une autre division altère en effet la supposée homogénéité des Canadiens français et des Canadiens anglais sur le plan de l'identification idéologique selon, justement, qu'on peut les dire conservateurs ou libéraux.

Chacun des personnages peut ainsi être situé sur cet échiquier à la fois culturel et politique. Du côté francophone, Father Beaubien et Marius Tallard représentent l'idéologie conservatrice - cléricale, ultramontaine et nationaliste - qui domine le milieu rural catholique. Côté anglo-écossais, la richissime famille des Methuens et l'homme d'affaires Huntly McQueen incarnent le pouvoir conservateur de la haute finance et de l'industrie, citoyens fidèles aux symboles de l'empire britannique; ce conservatisme peut aussi être relié, jusqu'à un certain point, à l'éthique protestante de l'église presbytérienne ${ }^{3}$. Du côté de l'idéologie

2. Dans ce contexte, humaniste et libéral désignent un discours où prédomine d'abord le sentiment d'appartenance du sujet à l'humanité et la reconnaissance de la liberté individuelle comme principe premier de l'existence. Position d'ouverture à l'autre - à la différence - en raison de cette mutuelle appartenance et reconnaissance, par-delà (mais, nécessairement avec...) l'ancrage identitaire dans la religion, la culture ou l'ethnie.

3. L'idéologie conservatrice catholique - ultramontaine et janséniste - ne serait pas sans affinités, selon Ronald Sutherland, avec le calvinisme écossais de type presbytérien ("The Calvinist-Jansenist Pantomime", Journal of Canadian Studies, Peterborough (Ontario), 5 mai 1970, p. 10-21). Marylin J. Davis a en effet montré l'incidence de ce discours puritain dans les romans de MacLennan ("Fathers and Sons", Canadian Literature, Vancouver, $\mathrm{n}^{\circ}$ 58, automne 1973, p. 39-50). Par ailleurs, Jacques Brazeau a souligné, dans "Perception du Canada français dans l'œuvre de Hugh MacLennan" (E. Cameron (éd.), Hugh MacLennan 1982, Toronto, Canadian Studies Programme, 1982, p. 35-47) que non seulement la vision du Québec rural y est simpliste, mais 
libérale, Athanase Tallard incarne le libre penseur francophone, lecteur de Voltaire et de Rousseau, à la fois aristocrate et républicain, ouvert sur la modernité; celle, surtout, qui vante le progrès. Son alter ego anglophone apparaît à la fois dans les personnages de Yardley, marin à la retraite, et de sa petite-fille, Heather Methuen. Enfin, Paul Tallard, fils d'Athanase et de Kathleen, irlandaise catholique, s'inscrit non seulement dans cette filiation libérale, mais incarne le drame des deux solitudes par sa double appartenance ethno-culturelle. Or le roman a justement pour enjeu la difficile assomption de cette double appartenance dans le contexte d'une critique du conservatisme québécois et canadien. Cette division interne de l'identité fondée sur l'idéologie a donc son importance puisqu'elle permet, par-delà la rigidité d'une opposition linguistique et confessionnelle, de frayer la voie à un discours de médiation susceptible de résoudre cette opposition. Car le roman n'est pas que mise en scène d'un conflit; il est aussi récit de son dépassement - en se faisant appel à la réconciliation, à l'unité, à l'harmonie —, et récit de fondation d'une nouvelle identité canadienne.

Pour ce faire, le roman affirme, en raison de ce discours humaniste et libéral, la primauté de l'expérience individuelle sur celle du politique (ainsi qu'on a pu le lire ici dans l'exergue); il prône également, sur cette base individuelle, un discours de réconciliation fondé, notamment, sur une certaine conception de l'amitié qui serait vérité première du rapport entre les sujets appartenant ou non à une même culture. En cela, le discours à l'œuvre dans le roman ne distingue pas l'amitié et la fraternité, dans la mesure où l'amitié relève davantage du domaine personnel, alors que la fraternité évoque l'idée de solidarité entre les peuples ou les diverses communautés politiques, réunis autour de la reconnaissance de l'égale dignité des sujets (individuels et collectifs) ${ }^{4}$. C'est donc l'ordre de ce discours sur l'amitié qu'il

qu'il n'y a aucune trace de politique provinciale dans le roman avec ce que cela suggère de tensions et d'ambiguiités. On pourrait ajouter que le politique ici est par excellence celui qui s'incarne dans le gouvernement fédéral et non dans le gouvernement provincial.

4. Dans sa déconstruction et lecture généalogique du politique, Jacques Derrida a montré que les concepts de l'amitié et de la fraternité s'inscrivaient notamment dans un discours familial et phallocentrique où la virilité de la vertu apparaît comme le noyau dur sur lequel se fonde le lien à l'altérité (voir Jacques Derrida, Politiques de l'amitié, Paris Galilée, coll. "La philosophie en 
138

convient d'abord d'analyser ici de manière à montrer quelle conception du politique et de l'écriture est à l'œuvre dans ce récit de fondation de l'identité canadienne.

\section{Yardley l'humaniste et le discours de l'amitié}

Outre Paul Tallard, le personnage clé de ce roman est sans doute le capitaine Yardley. C'est lui qui, par ses récits, réflexions et interventions, fonde le discours de l'humanisme libéral susceptible de dépasser le conflit inhérent aux idéologies conservatrices des deux cultures.

Ce discours apparaît au début du roman (chapitre 4) alors que Yardley raconte à Athanase Tallard comment lui, protestant anglophone parlant le français comme une vache espagnole, a pu s'établir sur une ferme au milieu d'une paroisse franco-catholique, à Saint-Marc-des-Érables. L'événement a certes quelque chose d'extraordinaire au pays des deux solitudes, ainsi qu'il le raconte lui-même avec la verve d'un vieux loup de mer sympathique. Le portrait quasi melvillien, dirait-on (ou proche de l'imaginaire de nombreux récits d'aventures en mer du XIx ${ }^{\mathrm{e}}$ siècle), sert ici de cadre au récit fondateur d'une certaine Amérique. Le récit du capitaine est d'ailleurs déterminant pour l'ensemble du roman et constitue un sorte de prologue qui donne le ton, en même temps qu'il fonde le discours moral du roman.

Dans ce fameux récit, Yardley raconte son amitié pour Luke Bergeron qu'il a naguère connu alors qu'ils étaient tous deux engagés à bord du même bateau. En manière d'ouverture aphoristique à son récit, Yardley déclare: "A sea-faring man keeps himself

effet", 1994). Disons, à cet égard, que ce que méconnaît le discours du roman de MacLennan sous le nom de fraternité, c'est plutôt un rapport à l'autre déterminé d'abord par la question de la reconnaissance. Cela dit, on entend ici par "fraternité" ce que Hannah Arendt, rappelant la leçon des Grecs, nomme l'amitié ou la philanthropia, qui "[...] se manifeste en une disposition à partager le monde avec d'autres hommes". Elle utilise le terme de fraternité pour décrire la solidarité qui apparaît chez les opprimés et les peuples parias; expérience de fraternité qui naît, dans ce cas, de la compassion ou de la pitié devant son propre malheur et celui des autres. Expérience cependant qui serait non pertinente pour fonder le véritable lien politique, celui de la philanthropia. Voir à ce sujet, H. Arendt, "De l'humanité dans de "sombres temps" ", Vies politiques, Paris, Gallimard, coll. "Tel", 1974, p. 1141. 
steady by thinking he's got a home some place ashore. But when he goes ashore for good, he generally finds the only home he's got is the friends he's made. And man, they're as like as not scattered all over the whole world" (p. 25). D'entrée de jeu, on note que la question de l'appartenance au sol ("land") comme insigne de l'identité est complètement relativisée par l'énoncé de Yardley. De son point de vue de marin — et donc de celui qui a fait l'expérience d'un espace apparemment sans frontières -, la question du "home" est plutôt, en effet, de l'ordre de l'illusion; le "home" véritable se trouve dans l'amitié (discours qui établit, on va le voir, la vérité du rapport à l'autre, à soi-même et à l'Histoire). Le vrai pays serait donc celui de l'amitié, par-delà l'appartenance à quelque identité ethno-culturelle et territoriale. De plus, et si la mer représente un espace sans frontières, n'est-elle pas le lieu où le sujet peut faire l'expérience d'une véritable liberté? Dès lors, le voyage en mer, symbole de liberté, peut devenir l'occasion de retrouver en soi-même les valeurs fondamentales, et ce, par-delà quelque inscription territorialisante. C'est ce jugement implicite, mais lisible comme une allégorie, que nous propose le texte. De même, le marin n'incarne-t-il pas, compte tenu de cette singulière expérience de la liberté (ou de l'espace), l'ouverture d'esprit que suppose la singularité de cette expérience? Expérience d'un espace ouvert qui engendrerait, par la force de quelque (merveilleuse?) causalité, un esprit libre et ouvert (bien que possible, cela n'est pourtant aucunement une condition nécessaire et suffisante). Ce jugement est encore implicite à ce stade du roman, mais déjà il s'impose plus ou moins subtilement pour construire peu à peu le discours moral du nouveau sujet canadien.

C'est donc depuis cette expérience qui transcende le discours de la territorialisation géopolitique que s'affirme la sagesse, sinon la légitimité, du discours de Yardley, sujet de l'ouverture qui peut ensuite parler avec probité de l'ouverture à l'autre. L'allégorie de ce voyage en mer est donc fort importante puisqu'elle s'oppose complètement au discours qui caractérise, dans le roman, les Canadiens français qui sont massivement identifiés au monde rural et au discours de l'enracinement, lequel suppose un esprit borné, sinon xénophobe. Par l'évocation de ce voyage en mer, une antithèse est ainsi posée, lourde déjà d'un certain jugement de valeur.

Après quelques digressions, Yardley poursuit son récit en nous racontant que c'est lors de l'un de ses nombreux voyages en 
140

mer qu'il a rencontré Luke Bergeron, un Canadien français originaire de Saint-Marc-des-Érables. Et, c'était dans l'espoir notamment de le retrouver - en vain, pourtant — qu'il a, dit-il, décidé de s'établir dans ce village. Cette rencontre entre Yardley et Luke aurait pu être tout à fait insignifiante ou banale, mais elle ne l'est pas en raison de certaines circonstances que Yardley nous livre ici comme un récit édifiant sur l'amitié :

Well, anyhow, [...] when Luke stepped onto the dock in Saigon he was a mighty surprised man, because outside the coolies all the white men talked French. He liked thet [sic]. And then one night ashore somme of those Frenchmen started riding Luke on the kind of French he talked. They said it was something awful to have to listen to - the same way Limeys used to make fun of the way I talk - and Luke got sorer and sorer, and then he lit into them. He was a mighty good man with his hands and he could use his feet like a lumberjack, but there was too many of those Frenchman, and me and Luke and the nigger, we got beat up so bad we couldn't lift ourselves off the floor when the cops came in. So they put us in the jailhouse (p. 26-27).

La toile de fond du roman étant celle du conflit que l'on sait, on constate que le récit de Yardley a une valeur hautement symbolique puisqu'il soutient en effet que l'intolérance à l'égard de l'autre peut exister au sein d'une même communauté là où l'accent produit des effets de discrimination à l'intérieur d'une même langue. C'est dire à quel point la langue n'est pas cet outil de communication idéalement neutre, mais plutôt, dans ce cas, l'instance à partir de laquelle s'actualise un discours de reconnaissance et un pouvoir de sujétion à l'égard de ceux considérés comme étant à la périphérie d'un centre ou d'une normalité (toujours plus ou moins fantasmatique, d'ailleurs). Le récit de Yardley relativise ainsi la sujétion - depuis la Conquête de 1759 - du Canadien français à l'Anglais, en montrant que l'ennemi ou l'intolérant n'est peut-être pas là où d'ordinaire on le croit. Notons aussi que devant l'accent de l'autre, Luke, tout comme Yardley, est sans préjugés, ouvert à l'autre et à l'amitié. Le récit a donc pour effet de s'appuyer sur un certain ressentiment envers la France, en même temps qu'il affirme, dans cette scène d'humiliation et de violence, une coupure avec le pathétique récit de l'abandon par la mère patrie. Or Yardley, qui parle avec un fort accent, est également victime de ce mépris. En cela, tous deux s'avèrent solidaires dans cette épreuve où ils sont prétendument inférieurs aux sujets de la métropole. C'est donc devant un tiers 
ennemi - le Français de France ou l'Anglais d'Angleterre - que vient se (re)fonder l'amitié entre Luke et Yardley, relativisant ainsi le conflit séculaire des deux communautés au Canada. Le mépris des autres à leur égard établit non seulement avec plus de solidité leur amitié, mais cette pénible expérience leur révèle aussi leur commune identité en face de cet autre, colonisateur et métropolitain. On se doute bien, dès lors, que cette commune identité, surgissant de cette épreuve, est aussi le gage d'une réconciliation à venir - question encore implicite toutefois à ce stade du roman - sur la scène politique canadienne. Le récit de Yardley s'inscrit ainsi dans le roman non seulement comme un récit de fondation, mais comme une promesse de réconciliation, laquelle façonne secrètement le cours de l'Histoire canadienne.

La morale de cette histoire, pourrait-on dire, est que si l'on est en conflit au pays, on se retrouve ami à l'étranger. Épreuve, à l'occasion de laquelle apparaît la noble force de l'amitié. Car, si Yardley et le "nigger" entrent dans une bagarre pourtant perdue d'avance pour défendre l'honneur d'un ami, c'est en définitive ce courage qui s'impose ici comme une noblesse du cour plus forte que les préjugés de race, de langue ou de classe. Le mépris et la violence n'auront donc pas triomphé de cette vertu, l'amitié; on peut même dire que, au contraire, elle l'aura révélée. L'autre - le Français ou l'Anglais de la métropole - est ainsi celui devant lequel deux sujets se reconnaissent être du "même": sujet-del'amitié par-delà ce qui les sépare sur le plan de la langue et les épisodes conflictuels de l'histoire politique québécoise et canadienne. Outre qu'ils sont tous les deux canadiens, force est de constater enfin qu'ils appartiennent de surcroît au vrai pays, celui de l'amitié. Ce qui suggère encore, plus ou moins subtilement, que le Canada est aussi — ou pourrait devenir — le pays de l'amitié, véritable et ultime fondement ici de l'identité politique; c'est en cela aussi que ce récit sur l'amitié s'avère non seulement le fondement moral et politique du roman, mais le récit de (re)fondation de la communauté canadienne.

La dimension fondatrice du récit de Yardley est lisible aussi en ce que cet événement se passe dans un ailleurs très éloigné: Saigon et la mer de Chine. C'est depuis cet ailleurs et cet avant que l'ici et le maintenant viennent se fonder dans un récit qui a l'aura de la fable ou de la légende, c'est-à-dire tout à la fois invérifiable et édifiant. Si nul n'est prophète en son pays, c'est sans doute que le sujet cherche à nommer sa singularité en se 
mesurant à l'altérité de l'autre, ou en prenant appui sur elle. C'est donc depuis l'étranger ou l'Autre qu'est posé le nouveau récit identitaire de la communauté canadienne: le récit se donne à lire comme un salutaire travail de décolonisation et ce, tant chez le Canadien français que chez le Canadien anglais. Toutefois, le récit de Yardley n'en opère pas moins un subtil détournement symbolique puisqu'il efface le discours proprement politique sur lequel se fonde l'antagonisme des Canadiens français et des Canadiens anglais pour en laisser prédominer la valeur individuelle de l'expérience dans le processus qui mène à la reconnaissance de l'autre. Ce récit a donc une valeur emblématique - sinon paradigmatique - dans le roman, en ce qu'il pose, à la base du rapport à l'autre, la prédominance du discours de l'amitié sur celui de la fraternité.

À la suite de ce récit, Yardley — incarnation du bel esprit empreint d'humanisme - pose à Athanase Tallard ce qui apparaît comme une ultime question de vérité: "One thing I'd like to ask you man to man, Mr. Tallard. I'm not a Roman Catholic. Does it make a hell of a lot of difference around here, not being a Catholic?" (p. 28). Point de chute de ce récit édifiant sur l'amitié, le capitaine interroge le présent, et plus exactement le bien-fondé du présent. Effet de contraste de ces deux temps, de ces deux paroles où l'on devine l'influence morale du récit légendaire de Yardley sur l'ici-maintenant. Cette question est en effet l'occasion d'un autre discours sur la tolérance et, comme tel, s'enchaîne parfaitement bien au récit du voyage en mer. Tolérance qui en appelle, pour discuter de la pertinence d'une identification confessionnelle, à une autre vérité: celle qui fonde, en l'occurrence, l'appartenance du sujet à l'Humanité. Cette appartenance ou identité première s'impose moralement à toute appartenance particulière. "Se parler d'homme à homme" ("man to man") est comme la nouvelle base d'un dialogue exempt de préjugés, posée depuis la reconnaissance de cette égale dignité humaine des sujets. C'est ce noyau dur de l'expérience et du savoir qui fonde ici le rapport à l'autre. Formule d'autant plus importante d'ailleurs qu'elle vient tout juste après la description ou le portrait des deux interlocuteurs: "Had he worn a Van Dyke beard he [Athanase] would have resembled Cardinal Richelieu. [...] In comparison, Yardley looked plain and workmanlike beside him" (p. 28). Entre le profil aristocratique de l'un et le profil ouvrier de l'autre, leur mutuelle appartenance à l'Humanité les fait égaux, par-delà ce 
qu'ils représentent. Plus loin, Yardley reprend d'ailleurs la même formule: "Man to man, Mr. Tallard, I put most of what I got into this land. I aimed to stay when I bought it. It's good land and it suits me" (p. 31). Cette expression a d'abord une fonction quasi phatique, marquant l'appel du sujet à la vérité et au sérieux; elle est là aussi comme le fondement de cette civilité qui, au-delà des différences, est gage de bonne entente. "D'homme à homme" s'impose ainsi comme ce qui, jusqu'à un certain point, supprime toutes les différences qui font obstacle à l'authentique rencontre de l'autre.

Cette fable sur l'amitié - et cette rencontre avec Athanase est d'ailleurs précédée par un autre récit édifiant, tenu cette fois par la voix narrative, et où l'on voit à quel point Yardley a l'esprit ouvert et sait s'adapter à son nouveau milieu (voir p. 20-21). Outre qu'on l'ait vu à la messe avec les francophones catholiques, Yardley a rencontré Father Beaubien et lui a donné de l'argent pour ses bonnes ouvres. De plus, il fréquente le magasin général, véritable place publique du village où il prend la parole (même si c'est dans un très mauvais français). Bref, Yardley incarne le bon citoyen, ouvert à l'autre, qui s'adapte et s'intègre en toute bonne foi. Ce qui ne l'empêche pas de rester fidèle à sa religion: "My old father, he whaled the Presbyterian catechism into me when I was a kid, so I'd feel mighty peculiar if I went permanent to another church" (p. 29). Ce qui ne l'empêchera pas non plus d'aller mourir sur sa terre natale, en Nouvelle-Écosse: "When a man's been born down there it stays his home no matter where he goes to live afterwards" (p. 300). C'est dire à quel point le personnage reste attaché, en définitive - et malgré son expérience de marin présentée d'abord comme celle d'un déracinement plutôt radical - à ses institutions et à son histoire. Il est ainsi décrit tout autant libre que fidèle, sans que l'on puisse savoir s'il y a là ambiguilté, contradiction ou véritable cohérence (cette incertitude étant plutôt comme le point de cécité du discours que véhicule le roman).

Cet épisode où se met en place le discours humaniste de l'amitié se termine par une proposition hautement symbolique: "Tell me, Captain. Do you play chess? I certainly do. I even got a set of men I picked up in India with elephants in place of bishops" (p. 31). Voilà l'invitation à la socialité du jeu, à l'amitié qui se noue autour d'un jeu plus que centenaire et qui représente la noblesse et la sagesse de l'honnête homme qui apprécie les 
144

choses de l'esprit. En mettant en scène une forme pour le moins intellectualisée de la confrontation et de la guerre, ce jeu permet de détourner, sinon de sublimer, la violence. Le jeu d'échec a donc valeur de symbole pour dire la domination des choses de l'esprit à l'égard du conflictuel et de la violence. En cela, il permet aussi d'établir le rapport à l'autre sur le mode du "fair play" alors que chacun se soumet aux règles du jeu dans un esprit de respect mutuel. Passe-temps de "gentleman" en somme qui s'accorde tout à fait avec les vertus civilisatrices de l'humanisme antique et moderne. La référence à l'Inde souligne également que ce jeu existe aussi dans les pays très éloignés (ou exotiques), ce qui lui confère ainsi valeur de sagesse universelle. Manière de communier autour d'un symbole de civilisation qui transcende l'identité ethno-culturelle. On constate donc que tout cet épisode du récit, et cette proposition qui lui sert en quelque sorte d'épilogue, a pour fonction de montrer comment un sujet se lie à l'autre en triomphant de la violence, des préjugés et du mépris.

Cette fable édifiante sur l'amitié que l'on vient d'analyser soulève cependant d'importantes questions quant à l'essence même du politique. Bien sûr, cet humanisme est en soi légitime; ce qui apparaît douteux toutefois, c'est de se servir de ce discours pour cacher une autre scène et un autre enjeu, politique celui-là, et qui implique l'histoire politique à la source de l'antagonisme des deux solitudes. Le recours à cet humanisme s'avère ambigu également dans la mesure où, soutenant le discours de l'amitié comme principe premier de la rencontre de l'autre, il méconnaît l'ordre du discours politique sur lequel s'établit le processus de reconnaissance entre les citoyens, les communautés et les peuples. En faisant ainsi primer la nature du lien individuel sur le collectif (politique), le roman donne une vision pour le moins biaisée de l'Histoire politique canadienne.

Poursuivons à cet égard le portrait du valeureux capitaine afin de mieux cerner le discours dont il est le porte-parole. Car nombreux sont en effet les épisodes où Yardley intervient pour faire une leçon de morale humaniste. On se rappellera à cet égard comment Frenette, le forgeron, (et sans doute presque toute la petite communauté réunie au magasin général) est tout à fait disposé à entendre, à propos de la guerre, une opinion contraire à la sienne: "From any other Englishman he would not have taken Yardley's words. But he knew the captain well by now and liked him and that made the difference. Yardley simply 
took it for granted that he was liked" (p. 63). La sympathie, sinon l'amitié, semble effacer les délicates et sensibles aspérités idéologiques. Comme si le sujet de l'énonciation (celui qui parle) l'emportait sur le sujet de l'énoncé (ce dont on parle), reléguant du même coup la rationalité dialogique - où s'exerce en principe la démocratie - dans les limbes plutôt suaves de la sympathie. Là encore, et bien qu'il soit évident que pour se faire un jugement l'on tienne compte aussi de celui qui parle, on constate que le débat proprement politique est tout simplement évité, le sujet se repliant du côté de l'affectivité dans son rapport à l'autre. On ne s'engage donc guère du côté du dialogue politique ainsi que nous le montre cette scène où Yardley, en désaccord avec un fermier qui évoque les conséquences d'une éventuelle défaite de l'Angleterre, lui rétorque avec fermeté que cela n'arrivera pas, sans toutefois qu'une argumentation véritable ne prenne forme. Yardley met simplement et aussitôt un terme à la conversation en affirmant qu'il faut respecter l'opinion d'autrui : "I don't blame you fellas for how you feel, the captain said. Guess I'd feel the same way if I was you. Only I'm not, so I don't" (p. 64). En fait, ce respect s'arrête pour ainsi dire sur une relative incommunicabilité de l'expérience et des idées. L'autre est reconnu dans son altérité, mais le dialogue tourne court, alors que c'est justement par la vertu du dialogue que des sujets peuvent éventuellement changer d'idée. Quelques pages plus loin — après l'épisode de la moustache coupée au "negro" (l'autre ami, avec Luke, de ces voyages en mer), lequel est l'occasion d'un autre récit édifiant sur le respect que l'on doit à l'autre - , le chapitre se termine en évoquant la paternelle amitié de Yardley à l'égard de Paul (voir p. 68). Ce noble sentiment tourne à la rêverie philosophique et à un autre discours sur la sagesse du capitaine-à-la-jambe-de-bois. La blessure de guerre contribue évidemment non seulement à l'aura d'héroïsme qui se dégage de sa personne (ce qui n'est pas en contradiction, il va sans dire, avec son humilité naturelle...), mais à camper le personnage du vieux loup de mer sympathique, conteur intarissable et sagace.

Trésor vivant de récits et de sagesse, Yardley sait bien que le savoir n'est que cette vanité qui voile l'insaisissable mystère du monde: "He was persuaded that all knowledge is like a painted curtain hung across the door of the mind to conceal from it a mystery so darkly suggestive that no one can face it alone for long" (p. 68). "Vanité des vanités, tout est vanité", disait déjà 
146

l'Ecclésiaste. Derrière le voile des savoirs apparaît vaguement quelque chose de vertigineux et d'abyssal que nul ne peut regarder en face sans en éprouver de la peur: "Of ultimate solitude he had no fear, for he never let himself think about it. But he knew that if he once started, fear would be there" (p. 68). Ce savoir du monde ne relève donc pas de quelque révélation abstraite, mais d'une expérience concrète ou empirique. C'est donc parce qu'il est d'abord le sujet d'une aventure ou d'un voyage au milieu de la nature qu'il connaît le mystère vaguement effroyable qui borde son expérience du monde. Yardley nous livre là une méditation sur l'origine qui prend encore la forme d'un récit allégorique alors que, par association d'idées, il se remémore une scène où, lors de l'un de ses voyages, il a pu observer: "Sharks and Barracuda moved in their three-dimensional element, self-centred, beautiful, dangerous and completely aimless, coming out from a water-filled cavern hidden beneath the promontory and slipping under the ship's keel [...]" (p. 69). Vision effrayante et fascinante de l'animale nature du monde à la fois belle et redoutable, cruelle et sans but. Manière de dire aussi que cette scène est le contraire de l'humanisme (de l'altruisme) considéré comme fondement du moins, en principe - de la culture occidentale. La méditation sur l'origine se termine d'ailleurs par cet épilogue: "[...] for people seemed so constructed that they were unable to use ideas as instruments to discover truth, but waved them instead like flags" (p. 69). Ce parti pris pour l'empiricité ou la concrétude de l'expérience va de pair, on va le voir plus loin, avec la condamnation des abstractions idéologiques (en particulier, du nationalisme). Cette rêverie philosophique est donc loin d'être innocente et s'inscrit plutôt dans la guerre des discours qui traverse le roman.

Cette rêverie montre le "naturel philosophe" de Yardley fondé sur l'expérience - au nom duquel il condamne l'intolérance, laquelle est toujours aussi une question d'ignorance (comme le disait déjà Platon). Yardley est donc, par excellence, le sujet de l'humilité, de l'humble savoir de l'expérience, celui qui possède la sagesse du sens commun, donnée à lui par l'observation de la nature, véritable savoir qu'on ne peut confondre avec un savoir purement livresque. Sa connaissance des livres est d'ailleurs celle d'un parfait autodidacte, gage peut-être ici d'authenticité de la lecture. On voit ainsi comment cette guerre des discours se construit peu à peu, par associations, passant d'une fable sur l'amitié à une scène allégorique concernant notre savoir 
originaire sur le monde. Ces scènes permettent de condamner, à la fin, le fanatisme d'un sujet drapé dans son idéologie.

Yardley est donc un sage qui ne cesse d'intervenir dans le cours du roman pour plaider la tolérance, le respect de l'autre. Ainsi l'épisode de l'excursion de pêche où il réprimande sa petite-fille Daphne parce qu'elle se moque de Paul qui se met à faire sa prière lorsqu'il entend sonner l'Angélus: "Thet's [sic] rude, Yardley said. Talking about other people's religions" (p. 164). De même, lorsqu'il reproche à sa fille Janet d'avoir dénoncé Marius à la RCMP pour que celui-ci soit enrégimenté: "It wasn't right, Janet, what you did. It wasn't a natural thing to do. Not all the wars in the world could make a thing like that" (p. 186). Yardley plaide ici la tolérance à l'égard des autres religions; il agit ainsi par amitié, semble-t-il, et par esprit de bon voisinage en condamnant la délation. Mais il escamote néanmoins la question de savoir s'il s'agit là d'un devoir que lui commande sa conscience. Ce discours égalitariste, il le tient encore à sa fille Janet qui a épousé Harvey Methuen, mort au champ de bataille et fils des célèbres Methuen. Pendant le défilé militaire célébrant la victoire des Forces Alliées, Yardley cherche à convaincre sa fille de s'émanciper de l'emprise de cette famille et, éventuellement, de sortir de son interminable deuil. Janet ne voulant pas l'entendre, le capitaine plaide encore l'égalité de chacun face au désir, à l'amour, à la solitude: "They're not so different [les Methuen], he said stubbornly. For thet $[\mathrm{sic}]$ matter, the way I see it, people aren't so different even from animals. Even the wildest ones can get mighty lonely. I think sometimes we imitate the animals in most we do" (p. 207). Au-delà des différences de classes, Yardley en appelle ici à la condition pour ainsi dire anthropo-biologique de l'humanite pour justifier l'égalité de tous du point de vue du sentiment. Cela suppose que ce n'est pas tant la culture qui détermine moralement le sujet, que son fond biologique, ce qui rejoint ainsi la morale empiriste, sinon anti-intellectualiste du roman, laquelle plaide alors les "faits" contre le règne de l'abstraction (qui est, dans le roman, celui surtout des guerres idéologiques de la première moitié de ce siècle en Europe).

À un autre moment, Yardley recommande encore la tolérance et l'ouverture d'esprit à Janet lorsque celle-ci s'oppose au mariage de sa fille, Heather Methuen, avec Paul Tallard:

Listen, Janet - One of the unfairest things we do in this country is to turn these religious denominations into flags. Why, thet 
148

boy's had all sorts of religion put onto him. He was a catholic, and then he was a Protestant, and then he was a catholic again, and between them they just about made a football out of him, but I know he's got a personnal religion of his own [...]. It's no more my business, or your business, than how a man makes love to his wife in his own bed after he's married to her. [...] A human being tries to be herself and you condemn her because she does! (p. 346)

La religion est donc présentée ici comme une affaire personnelle. Tout comme dans le cas de l'amour, tout sujet est d'abord libre de ses croyances et de ses convictions. L'amour traverse en cela les frontières identitaires, laissant prévaloir une autre loi, indifférente à ces inscriptions religieuse, civile et politique. L'amour, tout comme l'amitié d'ailleurs, est ici le véritable fondement de la relation à l'autre. Cette altercation avec Janet culmine d'ailleurs dans une longue tirade où Yardley juge sévèrement la morale conservatrice de son époque:

You talk to me about rebellion! I'm telling you something, Janet - the first word any child in the country hears to it is "No", and the first sentence he hears is "Be careful". [...] You and your friends - you go crazy if a girl and a boy make love to each other before they're married. But another twenty million people can get killed because our generation can't manage its own affairs and thet's not even immoral! (p. 347)

Ces quelques épisodes montrent bien que Yardley incarne l'humanisme libéral contre le conservatisme. Le capitaine souhaite enfin le mariage de Paul et Heather, malgré les deux "légendes" qui les séparent ("[...] victim of the two racial legends within the country"). Il bénit une union que sa fille Janet condamne au nom de sa fidélité au clan conservateur des Methuen. Or c'est justement contre la tyrannie de ces "légendes conservatrices" que ce mariage, acte symbolique de cette refondation du Canada, s'élève. Yardley prophétise ainsi, vers la fin du roman, l'avènement d'un "nouveau Canada" fondé non pas sur une vision conservatrice ou passéiste, mais sur une vision que l'on qualifierait de moderne où non seulement le progrès est en quelque sorte le moteur de l'Histoire, mais où l'expérience individuelle surplombe et dépasse les déterminations historico-politiques des sujets, peu importe qu'ils appartiennent à l'une ou l'autre des deux communautés fondatrices de l'État canadien: "Yet the country was changing. In spite of them all it was drawing together; but in a personal, individual way, and slowly, French and English getting to know 
each other as individuals in spite of the rival legends" (p. 301). Bien que légitime, ce jugement est cependant ambigu. D'abord, il apparaît fondé, puisque le sujet doit en effet pouvoir se libérer du discours idéologique qui pèse sur lui et sa communauté; l'expérience au niveau individuel lui permet de rencontrer l'autre dans sa singularité, sans le voile peut-être - car rien n'est jamais sûr en cette matière - de ses identifications (aliénations?) collectives. Mais ce discours a aussi quelque chose de pernicieux dans la mesure où il cherche à effacer le poids de l'histoire politique qui détermine aussi l'ancrage du sujet dans sa communauté et qui s'avère nécessairement marquant dans son rapport à l'autre. N'y a-t-il pas là, dans cet énoncé de principe, l'angélisme des bons sentiments qui gomme l'Histoire et la scène politique de la reconnaissance des communautés (linguistiques et culturelles)? C'est ce que pourtant la suite du roman confirme.

\section{Marius Tallard : l'enfant du ressentiment et du nationalisme}

Tout juste après cette fable pour le moins pédagogique sur l'amitié, le chapitre suivant dessine le portrait de Marius qui, à l'opposé de Yardley, incarne l'être de ressentiment, sinon le fanatisme du nationalisme canadien-français.

Le chapitre 5 s'ouvre sur la scène de l'intrusion clandestine de Marius dans le bureau-bibliothèque de son père, Athanase; il cherche alors à lui dérober de l'argent dont il aura éventuellement besoin pour échapper aux agents de la Conscription. Mais à cette occasion, il découvre le manuscrit secret du père, où celuici apparaît libre penseur, c'est-à-dire un critique sévère de la religion et du nationalisme. À cet égard, le père apparaît plus libéral que Marius, lequel se montre outré par de tel propos qui, selon lui, trahissent son peuple (voir p. 35). Scène de la vérité enfin dévoilée pour Marius qui constate ainsi l'hypocrisie et la trahison du père qui, en tant que député au parlement fédéral, est en principe tenu de dire tout ce qu'il pense. Voilà pourquoi, dans un premier moment et à ce stade du roman, Marius éprouve du ressentiment envers son père. Mais cette scène s'ouvre aussitôt sur une autre, passant du politique à l'espace personnel où se joue le désir. Refermant les tiroirs interdits, Marius se dirige alors vers la bibliothèque et saisit sur le rayon un livre d'art pour y contempler, peint par Titien, Botticelli, Ingres ou Rubens, les corps 
séduisants de femmes nues: "They signified only the female being he did not know, the being which was beautiful and dangerous and at the core of sin. His fingers shook as he turned the pages" (p. 36). Geste secret et coupable devant ce qui est pour lui l'angoissant mystère de la différence sexuelle. Ce livre de femmes nues, qu'il fréquente pour ainsi dire depuis longtemps, est en définitive porteur pour lui d'une interrogation lancinante à l'égard du désir, et dont il n'ose s'ouvrir à son père. La question de la féminité apparaît ainsi comme une impasse et un lourd secret en lequel Marius s'enferme: "Then, as always happened when he opened the book, he became afraid the pages would be marked by his fingers. [...] Not that his father would have cared. It was a matter of guarding his thoughts and essentiel self from others; this had become an obsession with Marius" (p. 37). Ce repli malsain sur soi-même, vaguement pathologique, ne peut que rendre suspecte la passion (faut-il ajouter, irrationnelle) avec laquelle, on va le voir, il s'est engagé dans le débat politique.

Cette scène débouche d'ailleurs sur une autre scène tout aussi symbolique de ses rapports difficiles avec le désir. C'est ce que nous apprenons en effet alors que, feuilletant le livre du péché, Marius est surpris par Kathleen, la jeune et séduisante épouse du père:

Ever since she had come to Saint-Marc nine years before, the house had seemed mysteriously evil, warm with sin. It was more than her beauty, more than the outrage he felt because his father had married a women young enough to be his own daughter. [...] It was the way she moved and sometimes the way she looked at him that gave her so much power over his senses. (p. 39)

Marius est évidemment troublé par cette femme qui incarne, à ses yeux de catholique rigoureux, la sensualité de la chair associée au péché. Cette sensualité enveloppe toute la maison et, par conséquent, s'oppose complètement à l'atmosphère dévote, sinon asexuée, créée par Marie-Adèle, sa défunte mère. Il éprouve en cela non seulement de l'agressivité envers Kathleen et son père, ainsi que nous le montre tout le dialogue de cette scène, mais se sent dépossédé ou maîtrisé par ce regard de femme: "Marius suspected that she saw through him completely and knew his secret thoughts as well as he did himself " (p. 40). Dans ses secrètes pensées il s'avère en définitive honteux de désirer ce qui est par excellence interdit, la femme du père: "The image of Kathleen's lush 
body still brimmed in his eyes and he felt sick from shame" (p. 41). Scène à l'évidence œedipienne, non résolue, et qui pèse prétendument sur toutes les facettes de la personnalité de Marius. Or c'est exactement ici que se construit dans le roman une causalité en vertu de laquelle la scène honteuse de l'impasse oedipienne cherchera à se résoudre sur la scène politique: "Thoughts of the army filled him with dread, mixed with bitterness against the English who were forcing the evil of war upon him. And the dread and the bitterness served to cancel out his shame" (p. 42). Une scène en cache ainsi une autre où le sujet n'ose affronter semble-il ses véritables conflits. Mais avant de clore l'analyse de cette scène avec Kathleen - où apparaît cette superposition biaisée du familial et du politique - , il convient de creuser davantage le sens de cette impasse œdipienne en cernant au plus près le personnage de la défunte mère de Marius, Marie-Adèle.

Marie-Adèle nous apparaît d'abord dans ce portrait : "The woman's eyes were lowered as though in modesty before the camera. It was the virginal face, almost the nun's face, of his mother "(p. 41). Le contraste est éloquent entre la sensualité démoniaque de l'une et l'humilité toute virginale de l'autre. Marie-Adèle est donc mère et, cependant, symboliquement vierge. Décrite comme une sainte nitouche, elle serait morte prématurément à force de dévotions envers la Vierge. Mariage pour le moins ambigu, en effet, où le désir est refoulé pour mieux être sublimé sur la scène religieuse de l'adoration. Pas étonnant dès lors que Marius ait du mal à symboliser sa place en tant que sujet du désir, angoissé qu'il est notamment par la question de la différence sexuelle qui, d'une certaine façon, lui a été mal transmise.

Cela, nous l'apprenons peu à peu au cours du roman alors que nous est révélée la nature du lien qui unissait Athanase et Marie-Adèle. Mariage d'emblée malheureux puisque le père s'était marié sans éprouver de désir: "It was a bitter piece of irony that in marrying Marie-Adèle he had chosen the one women he then knew who could feel no sexual attraction for him whatever" (p. 76). C'est de cela dont Marius est l'héritier: enfant d'un mariage raté, fondé sur l'erreur ou l'inconsciente volonté de se tromper. Mariage presque blanc alors que sa mère passe ses jours à prier: "Even before the birth of Marius she had estranged herself from him, and after Marius was born she acquired a peculiar absorption in prayers and visions. Gradually her religion had become her whole life" (p. 76). Cette mère semble se retirer dans 
152

la prière pour ne pas avoir à assumer son devoir d'épouse (ou l'amour qu'elle ne ressent peut-être pas, bien que cela ne soit pas thématisé). Marius est ainsi l'enfant que sa mère abandonne symboliquement pour mieux se dévouer à Dieu. De ce passé, Athanase a évidemment gardé un souvenir amer: "He had found many and he remembered charming moments with them, but on the whole he bitterly regretted this period of his life as a time of waste" (p. 76). Un certain ratage pèse donc sur la relation entre le père et le fils, non-dit ou secret qui assombrit leur relation :

He often wondered how much Marius knew. The boy worshipped his mother's memory. Athanase sighed. There was a mystery too deeply rooted in his relations with Marius for him to comprehend its full meaning. He had loved the boy. He still did, but apparently love was inadequate where there was no understanding. (p. 77)

Ce ressentiment, plus loin, prend la forme d'une accusation: "She was a saintly women. She suffered unspeakably because of you, because of your sins and wicked thoughts and your sneers at religion" (p. 168). Mais l'on devine que derrière ce reproche s'en cache un autre, plus ou moins avoué, à l'égard d'un père qui n'a pas désiré la mère; de là, sans doute, le ressentiment de Marius envers son père et la vie en général. Or cette histoire n'est pas seulement celle d'un désir raté, elle est lourde aussi d'un événement secret qui a eu lieu lors de la mort de Marie-Adèle. Ce secret apparaît inexplicable, noué aux mystères profonds du désir: "How explain to an ascetic what had happened the night MarieAdèle died? How explain to anyone? How tell even himself that there is any logic in human life after such a night?» (p. 168). Est révélée ici la scène secrète qui hante la conscience du père. Athanase aurait en effet quitté sa femme agonisante pour se réfugier dans les bras de celle qui deviendra sa femme:

Then he could stand it no longer. He groped like a blind man to the door. He walked for hours. [...] Finally he remembered Kathleen and went to find her. [...] He looked at her and she held his eyes, and with an understanding as simple as a child she gave herself to him that night. He cried himself to sleep in her arms and she lay awake holding him (p. 169). [...] So Marius had known! So this was the thing that had lain between them all these years! (p. 170)

Ce geste odieux ou moralement obscène hante le père, le culpabilise à l'égard de son fils qui, tout en ne sachant rien sans doute de cette scène secrète, l'incrimine cependant du regard parce 
qu'il est d'abord le fils malheureux d'une union que son père n'a jamais vraiment assumée. Voilà donc ce que nous invite à lire le roman. Marius est d'abord un enfant malheureux, et c'est essentiellement par ce trait de caractère que s'explique son engagement sur la scène politique. Cela apparaît clairement lorsque son frère Paul, plus tard, juge l'engagement politique de celui-ci: "Would she understand that Marius finished himself the moment he began blaming everyone else for what he lacked himself?" (p. 298) La causalité est ainsi posée où l'on voit que Marius éprouve du ressentiment envers le monde - les Anglais, la situation politique des Canadiens français - pour mieux refouler l'autre scène, familiale, d'où provient sa frustration et sa haine.

Voilà le sombre héritage familial qui ronge Marius. Réfugié dans sa chambre après son altercation avec Kathleen, Marius se recueille devant un petit autel et le portrait virginal de sa mère, refoulant sa honte (son désir) à mesure qu'il nourrit son ressentiment, sinon sa haine, pour l'Anglais (voir p. 41-42). Mais alors que cette scène avec Kathleen nous a révélé un jeune homme fragile, sombre et perturbé, la scène suivante nous le montre au contraire comme un orateur éloquent, passionné et charismatique:

Marius had no idea how he was doing this, nor even what he was saying. His own unhappiness and frustration had been feeling up a well inside him for ten years and now he felt he could go on speaking out of it forever. The listening crowd leaned forward and gave him back a mysterious elation in exchange for his words. They loved each other, Marius and all these strange people (p. 45).

Telle est en effet la scène sur laquelle s'ouvre le chapitre 6, tout de suite après celle de l'impasse œedipienne. Un lien de causalité est donc ici construit alors que nous passons de l'espace familial à l'espace public. À l'occasion d'une assemblée politique contre la Conscription où il prononce un discours, Marius apparaît comme un possédé, un exalté, un fanatique qui, pour l'essentiel, puise ses paroles dans sa profonde frustration personnelle. La foule, séduite par le discours de Marius et communiant mystérieusement avec lui dans l'amour, apparaît nécessairement manipulée par une passion dangereuse et néfaste, puisque Marius n'est pas animé par l'amour, mais bien par la haine. Leçon de chose au passage sur le leurre des passions et qui rejoint, plus loin, le discours de son demi-frère Paul sur la nécessité d'être pragmatique, c'est-à-dire mesuré et raisonnable face au politique. Cela dit, 
154

on constate que le ressentiment de Marius relève moins de l'analyse politique que de la scène douloureuse et obscure de sa petite histoire. Cette scène lui revient d'ailleurs, au fil du roman, comme une angoissante question sur les véritables motivations de ses gestes et paroles: "[...] he asked himself if he really did hate the English, and why. By nature suspicious, he was suddenly suspicious even for himself. The face of Kathleen rose before his mind. But his hatred was caused by more than one women alone. He had always hated them" (p. 180). Là encore, le texte est on ne peut plus explicite sur le fait que la haine de l'Anglais cache en définitive la haine des femmes. Cette scène de vérité, qu'il n'a jamais su affronter, concerne d'abord, ainsi qu'on l'a vu, sa propre relation malheureuse à sa mère, Marie-Adèle. La petite histoire apparaît donc ici, vérité de l'Histoire.

De la scène de l'amitié (chapitre 4), en passant par celle de l'CEdipe malheureux (chapitre 5), on aboutit ainsi à celle du fanatisme politique (chapitre 6). La scène de l'CEdipe et celle du fanatisme politique s'opposent, au fil de la lecture, dans une relation de causalité, par le montage formel du roman, alors que ce qui se joue sur la scène politique est liée structuralement à la scène précédente. Ruse du roman où la scène de l'CEdipe et celle du ressentiment se succèdent pour produire, par métonymie, l'apparente rationalité d'une causalité; le roman nous invitant à voir dans une scène oedipienne la causalité originaire de cette adhésion de Marius au nationalisme conservateur — aux forts accents de fanatisme - du Canada français.

L'impasse odipienne qui pèse sur la vie de Marius fait donc en sorte qu'il appréhende la scène du politique comme une scène tout à la fois d'affirmation et de compensation, celle-ci servant d'exutoire à son conflit familial. Le roman construit ainsi cette opposition afin que l'on juge d'un discours politique pour, évidemment, le disqualifier. Nationaliste fanatique et anglophobe qu'outrage la politique du gouvernement canadien en faveur de la Conscription (Première Guerre mondiale), Marius tire en réalité toutes les motivations à l'origine de son engagement politique d'une causalité essentiellement psychologisante, comme s'il ne pouvait y avoir de raisons proprement politiques pour s'opposer à la légitimité de la Conscription ${ }^{5}$.

5. Dans son roman Return of the Sphinx (Toronto, MacMillan, 1967) - où est abordé la question du terrorisme au Québec dans les années soixante - 
Bien sûr, nous sommes ici dans l'espace imaginaire du roman, et il est entièrement plausible qu'un sujet puisse jouer la scène de ses conflits personnels sur celle du politique. Mais, si l'on considère qu'il s'agit ici d'une sorte de roman à thèse où d'emblée se dessine une guerre des discours politiques, on ne peut que constater à quel point la vision du nationalisme canadien-français est monolithique, ne tenant aucunement compte de la complexité de cette question sur le plan de l'Histoire. Certes, il ne s'agit pas non plus d'opposer abstraitement le sujet du politique au sujet du désir, tant il est vrai que le sujet du politique est aussi travaillé par son désir, cela pouvant en effet le conduire à toutes sortes de dérapages. Mais il est vrai aussi que la scène du politique a sa rationalité propre où, par-delà la contingence ou la finitude du sujet qui s'y engage, la justice et le droit cherchent à s'imposer pour le bien commun. Le politique se situe en cela au carrefour de l'individuel et du collectif, et il n'y a peutêtre en définitive que la probité (et le désintéressement personnel) qui fait qu'un tel engagement ne dérive pas du côté du cynisme, d'une folle passion ou de quelque fanatisme. Toutefois, le roman ne s'embarrasse pas de ces distinctions et disqualifie le bien-fondé des résistances des Canadiens français à la Conscription en faisant glisser les causes de cette résistance du côté du roman familial. Il démonise, en l'occurrence, le nationaliste canadien-français ${ }^{6}$.

MacLennan répète non seulement sa critique du nationalisme, mais tend encore à expliquer l'Histoire par le roman familial. Ce qui n'est pas le cas du roman de Leonard Cohen, Beautiful Losers qui, traitant aussi du terrorisme, rejoue cependant la dimension symbolique du légendaire et de la monumentalisation à l'œuvre dans l'Histoire (ainsi que j'ai pu le montrer dans mon article, "Tombeau de Catherine Tekakwitha. Histoire, deuil et prière dans Beautiful Losers de Leonard Cohen ", Théologiques, vol. 5, n 2, octobre 1997, p. $107-126$.

6. Rappelons que dans cet épisode où Marius prononce son discours politique, celui-ci a été appelé à remplacer le flamboyant orateur qu'était Henri Bourassa, rien de moins (voir p. 46)! Un lien est ainsi posé entre l'une des figures marquantes du nationalisme canadien-français de l'époque et Marius, l'être de ressentiment. Voici ce que déclarait toutefois Henri Bourassa en 1904 à Jules-Paul Tardivel: "Notre nationalisme à nous est le nationalisme canadien, fondé sur la dualité des races et sur les traditions particulières que cette dualité comporte. Nous travaillons au développement du patriotisme canadien, qui est à nos yeux la meilleure garantie de l'existence des deux races et du respect mutuel qu'elles se doivent. Les nôtres, pour nous comme pour M. Tardivel, sont les Canadiens français; mais les Anglo-Canadiens ne 
Plus loin dans le roman, c'est au tour de Paul de faire le procès du nationalisme de Marius. Lui aussi considère alors le fanatisme de son demi-frère comme le résultat d'une impasse individuelle :

Does she [Heather] realize that the whole trouble now is that everyone is trying to make the facts fit their feelings instead of making their knowledge fit the facts? [...] Would she understand that Marius finished himself the moment he began blaming everyone else for what he lacked himself? In Marius's mind, those who don't agree with are traitors. What his theories add up to is as crude as voodoo. But if they shut you out everywhere, what do they expect to get but voodoo. For four years the depression has been screaming at people to surrender and go into the woods together and beat the tom-tom. What has happend in Germany is only what happens to any single man when he lets what matters spill out. So now there's a gigantic involuntary conspiracy to make everyone surrender in the name of everyone else, in the name of somme abstract idea (p. 298).

sont pas des étrangers, et nous regardons comme des alliés tous ceux d'entre eux qui nous respectent et qui veulent comme nous le maintien intégral de l'autonomie canadienne. La patrie, pour nous, c'est le Canada tout entier, c'est-à-dire une fédération de races distinctes et de provinces autonomes. La nation que nous voulons voir se développer, c'est la nation canadienne, composée des Canadiens français et des Canadiens anglais, c'est-à-dire de deux éléments séparés par la langue et la religion, et par les dispositions légales nécessaires à la conservation de leurs traditions respectives, mais unies dans un attachement de confraternité, dans un commun attachement à la patrie commune". Texte cité par P.-A. Linteau, R. Durocher, J.-C. Robert, Histoire du Québec contemporain, tome 1: De la confédération à la crise, Montréal, Boréal/Compact, 1989, p. 704. Voilà, à l'évidence, un discours qui ne se fonde pas sur la haine, mais sur la reconnaissance et le respect mutuel. Outre Henri Bourassa, faut-il rappeler que Wilfrid Laurier, ex-premier ministre du Canada (et fédéraliste, bien sûr), fut également contre la Conscription (voir J. Lacoursière, J. Provencher, D. Vaugeois, Canada-Québec. Synthèse historique, Montréal, Édition du Renouveau pédagogique, 1978, p. 481)? C'est là sans doute le point de cécité du roman que de ne pouvoir poser un personnage qui soit à la fois nationaliste et fédéraliste (le nationalisme étant ici démonisé). Rappelons enfin qu'après cet épisode où Marius prononce son discours, la voix narrative épilogue une fois de plus sur les vertus d'une politique de l'amitié ou de la reconnaissance strictement individuelle: "Plenty of English and French-Canadians worked together in stores and in factories and got on with each other. He [Marius] didn't like it; he would teach his people to hate the English the way he hated them. The fact that he knew no English-Canadians well except his stepmother didn't enter his thoughts" (p. 53). 
On constate que Paul critique l'idéologie de son époque comme étant celle d'une régression tribale, contraire au vaste mouvement civilisateur de l'Occident dominé par l'indissociable triomphe de la raison et de l'individualisme ${ }^{7}$. Le désœuvrement, le désarroi, la fuite dans l'abstraction idéologique entravent prétendument l'individualité du sujet, qui se perd alors dans de sombres et violents mouvements de masse. Paul juge aussi Marius depuis cette faillite idéologique de l'Occident, puisqu'il considère le nationalisme de celui-ci comme un refus de la modernité, celle d'une vision empirico-pragmatique qui, justement, ne s'appuie que sur les "faits". Madame Rocheleau — représentant par excellence la mère canadienne-française - incarne cette lucidité du jugement qui ne donne foi qu'aux faits et non au fanatisme idéologique d'un Marius: "She has a respect for facts, he said. [...] If this country were invaded and the Germans were ten miles away and it was time to pick the potatoes, she'd be out picking them. [...] Are there many like Madame Rocheleau in Québec? So far there have been enough to guarantee that my brother remains an unsuccessful politician" (p. 361). De plus, le nationalisme de Marius ne s'oppose pas seulement au discours des "faits", mais également à l'idéologie moderniste du progrès scientifique:

He kept repeating the same things over and over. Economics? What did economics matter? A pure race, a pure language, larger families, no more connection with the English, no interference from foreigners, a greater clerical control over everything - with these conditions Quebec will reach the millennium. Scientists could split the atom and circumnavigate the globe in a week, but Marius had no difficulty reducing everything to race, religion and politics. [...] With every sentence he uttered, Marius was binding the strait-jacket tighter and tighter around himself. Was this the same process he had witnessed in

7. Cette critique du nationalisme comme régression tribale rejoint celle d'un Pierre Elliot Trudeau (Le fédéralisme et la société canadienne-française, Montréal, Hurtubise-HMH, 1967) qui, en contrepartie, fait l'apologie de la rationalité de l'État (alors que le roman de MacLennan valorise plutôt une politique que l'on a dit être celle de l'amitié). Si cette méfiance à l'égard du nationalisme peut légitimement se comprendre, suite au nazisme, il n'empêche que ce jugement sur l'Histoire escamote la singularité de la situation québécoise. Dans son livre, Nationalité et modernité (Boréal, Montréal, 1998), Daniel Jacques a remis en question l'équation entre nationalisme et intolérance en montrant que la nation n'est pas nécessairement l'antithèse de la démocratie et de la liberté. 
158

Europe? If so, Marius didn't know it. To try to handle science by nationalism! God, did they have to do that here as well? It seemed as though Marius had to bind others to make himself free (p. 374).

Le nationalisme de Marius est ainsi posé comme une régression à l'égard de l'évolution progressiste et moderne de la science et de l'industrialisation. Là encore, son repli nationaliste ne serait que la scène inverse de celle qui se cache dans l'intimité de sa petite histoire, entraînant de surcrôt toute la communauté dans les eaux troubles de son propre malheur. C'est aussi ce discours qui sous-tend le nouveau récit identitaire canadien conçu comme dépassement de quelque patriotisme enfantin: "I suppose patriotism was originally nothing but the remembrance of childhood. Childhood is always magical" (p. 362). Une hiérarchie est ici posée où la modernité s'oppose clairement au nationalisme, comme l'adulte à l'enfant, l'homme de raison à la pensée magique de l'homme primitif. Ce qui suppose, enfin, que le nouveau récit identitaire canadien se fondera sur cette rationalité moderne, magiquement immunisée contre les dérives de son propre nationalisme. On note également dans le passage cité plus haut que Paul a une conception du politique où s'affirme la primauté de l'expérience des faits sur l'abstraction idéologique - sur l'idéalisme, l'intellectualisme - , ainsi qu'il le dit à Heather: "I don't seem able to look at politics as if it were a science. I look at people instead" (p. 312) ${ }^{8}$. Certes, on peut soutenir que la politique n'est pas une science, mais cela ne veut pas dire qu'elle n'est pas la scène où s'exerce une certaine rationalité susceptible de régler les différends qui surgissent dans la société. La position individualiste de Paul serait plutôt une position politique qui s'ignore (ou tente de le faire) et qui, par là, cherche à se légitimer sur une nature ou une évidence. Par son appel à la concrétude de l'expérience, le discours de Paul sous-entend que si le nationalisme des Canadiens français est une sorte de régression tribale, le nouveau récit fondateur de l'identité canadienne serait en quelque sorte au-delà du politique, et donc du discours de reconnaissance qui

8. Hugh MacLennan assume le même discours que son personnage lorsqu'il dit: "I am no sage and no political scientist and I profoundly mistrust abstractions when they are applied either to human beings or to what Dr. Camille Laurin calls la collectivité" ("Two Solitudes: Thirty-three Years Later", The Other Side of Hugh MacLennan. Selected Essays Old and New, édition d'E. Cameron, Toronto, MacMillan, 1978, p. 294). 
règle les rapports entre les communautés. L'appel aux "faits" est donc là encore une autre ruse du discours qui permet au sujet de sortir du politique pour faire prédominer ce qui se présente comme une sorte de "nature objective" (en opposition aux abstractions idéologiques). Cette position n'est elle-même pas sans ambiguités et cache son propre terrorisme intellectuel, sinon son intolérance.

Après avoir rencontré Marius pour une dernière fois, Paul prend non seulement conscience de l'erreur de celui-ci, mais aussi de la lucidité (politique) que lui donne la singularité de sa place comme enfant issu d'un mariage mixte:

Out of the society which had produced and frustrated him, which in his own way he had learned to accept, he knew that he was at last beating out a harmony. His fingers seemed to be feeling down through the surface of character and action to the roots of the country itself. In all his life, he had never seen an English-Canadian and a French-Canadian hostile to each other face to face. When they disliked, they disliked entirely in the group. And the result of these two group-legends was a Canada oddly naive, so far without any real villains, without overt cruelty or criminal memories, a country strangely innocent in its groping individual common sense, intent on doing the right thing in the way some children are, tongue-tied because it felt others would not be interested in what it had to say; loyal, skilled and proud, race-memories lonely in great spaces (p. 377).

On constate que le jugement qu'il pose sur l'histoire canadienne laisse prédominer l'expérience individuelle sur la dimension collective. Car, "face to face", il n'y a pas de conflit; ce n'est prétendument que par quelque fanatique identification à leurs "légendes" - aux récits conservateurs de chacune de leur tradition - que l'affrontement des deux communautés a lieu. À ce niveau, le roman disqualifie encore tout le discours historicopolitique sur lequel se fondent les tensions constitutionnelles depuis la Conquête de 1759. Vision somme toute idyllique où le Canada apparaît, en son fondement, paré de tous les bons sentiments de l'humanisme libéral pour mieux cacher, toutefois, la violence physique et symbolique sur laquelle est malgré tout établi le pays. Violence entre conquérants européens qui s'est faite, on le sait, après la violente sujétion des peuples amérindiens. Oubliant cela, il cède à une vision plutôt angélique où domine prétendument la droiture et l'innocence. 
160

Cela dit, cette belle identité vertueuse est encore, semble-t-il, mal assumée puisque le Canada ne serait encore... qu'un enfant. Immaturité que veut justement, par sa vocation d'écrivain, dépasser Paul, fort de sa supposée lucidité politique et culturelle. Si Paul est en effet déchiré entre deux cultures, il est pour ainsi dire le troisième terme qui résout l'opposition initiale entre les deux cultures $^{9}$. Il dépasse apparemment toutes les oppositions du roman en étant catholique et protestant, francophone et anglophone, successivement riche et pauvre. Il incarne le personnage emblématique de la réconciliation et de la nouvelle identité made in Canada. Mais on ne peut s'empêcher de constater à quel point ce destin est exceptionnel, et qu'il est, sans doute, tout à fait idéal de le présenter comme point de repère d'une nouvelle fondation du récit identitaire québéco-canadien.

Du conflit des deux solitudes naît malgré tout la musique d'une secrète harmonie que Paul sait entendre depuis la singularité de sa place, dans cet apparent entre-deux culturel qui le rend censément lucide. Fond harmonieux qui s'impose lentement pardelà le conflit des deux solitudes et que Paul voudra révéler en tant qu'écrivain. C'est dire que sa vocation d'écrivain se précise et s'affirme en même temps qu'il veut occuper le centre de ce conflit dont il saura éventuellement dénouer le drame. En cela, Two Solitudes se fait aussi le roman de la promesse du Livre à venir susceptible de raconter l'avènement de cette voix canadienne

9. C'est le discours utopique de ce roman que de supposer ce métissage parfaitement réussi, laissant ainsi dans l'ombre la question de la reconnaissance des sujets et de leurs langues, alors que la situation politique qui est la leur n'est pas fondée sur l'égalité. En cela, si le roman cherche à dépasser un certain discours traditionnel qui voyait dans le mariage mixte le spectre de l'assimilation - souvenons-nous de L'appel de la race de Lionel Groulx - , il verse cependant dans une sorte d'angélisme alors qu'il ne considère pas, sur le plan identitaire, les enjeux symboliques qui rattachent un sujet à sa langue. Question que j'ai abordée depuis l'analyse d'un roman de Clark Blaise, un écrivain anglophone d'origine francophone, et donc assimilé ("Traduire son nom en passant la frontière. La quête identitaire dans Resident Alien de Clark Blaise ", Le texte et le nom, Martine Léonard et Élisabeth Nardout-Lafarge (dir.), Montréal, XYZ, coll. "Documents", 1996, p. 181-194). Ce discours de l'amitié et d'un certain métissage canadian n'a d'ailleurs pas séduit, loin de là, un Jacques Ferron dont le récit Les confitures de coings (précédemment La nuit) met justement en scène, d'une part, le leurre d'une amitié (avec le fameux Frank Archibald Campbell) qui achoppe sur la question politique et, d'autre part, un autre processus identitaire où le sujet métissé (enquébécquoisé) a pour point d'ancrage son identification ou son désir de la langue. 
émergeant enfin des ténèbres. Mais d'où vient cette harmonie promise, agissant secrètement dans le cours de l'Histoire?

\section{La secrète musique de l'Histoire (épilogue)}

So the country brooded on through midsummer, each part bound to the others like a destiny, even in opposition forming a unity none could dissolve, the point and counterpoint of a harmony so subtle they never guessed its existence. (p. 155)

Ainsi se termine le chapitre 16, laissant là, pour un temps, le récit de la discorde nationale. La voix narrative semble en effet se lever au milieu de ce conflit, drapée dans sa sagesse, pour épiloguer sur le drame passé et révéler qu'au-delà de cette superficielle dissonance due à quelque discorde politique, il existe ce fondement harmonieux qu'une oreille musicale devrait savoir reconnaître comme la secrète ou subtile musique d'une même œuvre, garante de l'unité identitaire du sujet canadien. Non pas deux partitions où se donnerait à entendre l'hétérogénéité de ces voix sur la scène politique, mais une seule partition absorbant et résorbant peu à peu toute dissonance pour laisser triompher l'harmonie. Lovée au fond de l'Histoire, cette secrète harmonie ne se révélerait toutefois que lentement à qui sait l'entendre ou la faire entendre. L'harmonie est donc posée là comme la vérité de l'ancrage historique de tous les sujets, par-delà les enjeux politiques inhérents à la reconnaissance de leurs différences. Elle est comme le troisième terme de cette opposition politique au nom de laquelle l'hétérogénéité des communautés sera surmontée.

Le roman porte ainsi, revêtue de la magique autorité de la troisième personne du singulier - voix originaire du récit, voix tutélaire de l'Histoire - , cette voix annonciatrice de la réconciliation. L'harmonie se révèle dès lors par cette voix narrative, omnisciente et omnipotente, qui livre, aux détours des descriptions et de l'intrigue, les vérités sur lesquelles sont fondées - pour être résolues - les dissonances qui traversent l'Histoire. Cette voix narrative intervient donc dans le cours du roman pour affirmer que, ce qui peut nous apparaître comme une lutte et un continuel affrontement entre francophones et anglophones, n'est en fait qu'un drame ponctuel, soumis à la vérité d'un long processus historique ou à une ruse de l'Histoire (pour le dire comme Hegel) 
au nom de laquelle triomphera cette unité et cette harmonie, pareille en cela au projet enfin accompli de quelque suprême providence. Le discours, malgré sa neutralité apparente, se fait promesse de l'avènement triomphant d'une identité historique et nationale.

Cette voix narrative, posée en tiers, fonctionne ainsi dans le roman comme une sortie du cadre dialogique inhérent à la scène du politique. Elle se situe d'emblée au-dessus du débat, sans visage et sans nom, pour dire la nature et l'issue d'un conflit et, ce faisant, l'arbitrer et le dominer depuis un autre savoir. Ce narrateur omniscient qui prophétise l'harmonie est donc déjà le dispositif d'un récit qui nie jusqu'à un certain point le politique comme discours susceptible de déconstruire son propre lieu d'énonciation par la raison critique, qui ne cesse d'interroger, en effet, le bien-fondé qui l'anime. Scène où s'affirme ouvertement - en principe - le fondement de l'action du sujet dans l'Histoire. En cela, la scène du politique révèle également que le sujet de l'énoncé est inséparable du sujet de l'énonciation, puisque c'est aussi la probité de celui qui parle qui cautionne, en bout de ligne, la validité de la parole publique. Voilà pourquoi on peut dire que cette voix qui plaide l'harmonie parle et pourtant s'efface, dans le même geste, pour échapper à la finitude qui détermine le sujet du politique. Elle répond du sens de l'avenir de l'Histoire canadienne, mais elle n'est plus là dès lors qu'on l'interpelle. Cette voix séduisante de l'harmonie prend aussi le masque des bons sentiments, gommant le politique, scène du litige, du différend, du tort ou de toutes autres formes de conflits soumis, en principe, à la finitude de toute situation dialogique. Le discours de l'harmonie suppose ainsi le montage discursif et narratif de cette voix invisible, idéalement neutre, qui résout les conflits et fait avancer l'Histoire et son sujet. C'est pourtant par la ruse de cette voix qu'est posé, à l'égard de l'Histoire, un type de causalité que le lecteur, plus ou moins leurré, voudra lire comme une motivation psychologique ou morale.

Que l'on évoque enfin le politique en terme musical n'est pas, en soi, un dérapage idéologique. Faut-il le rappeler, la métaphore ou l'allégorie ne sont pas d'entrée de jeu des images fallacieuses voilant quelque nue réalité. Cela dépend plutôt de la manière et du contexte - c'est-à-dire de l'énonciation — depuis lesquels ce discours est construit. Or cet appel à l'harmonie musicale s'inscrit dans un discours où la spécificité du jugement politi- 
que se trouve arraisonnée à un autre savoir et à une autre vision de l'Histoire. Ainsi, ce n'est certainement pas la même chose, parlant du politique, d'en appeler à l'unité et à l'harmonie plutôt qu'à l'égalité: dans le premier cas, cela suppose un discours où l'identité (à venir) du sujet canadien est fondée sur une vision psychologisante de la culture et de la politique, alors que, dans l'autre cas, la relation entre les deux communautés se règle plutôt sur le discours de la reconnaissance politique et constitutionnelle. L'appel à l'égalité des communautés ou des peuples implique notamment la reconnaissance de cette hétéronomie, laquelle peut ensuite être reconnue dans quelque texte constitutionnel. De là, il n'est pas interdit de parler de relation (musicalement) harmonieuse sur cette base politique ${ }^{10}$.

Lorsque cette voix narrative se tait, le discours de l'harmonie revient parfois par la bouche de Huntly McQueen, l'homme d'affaires écossais et presbytérien qui incarne le Chevalier de la Finance canadienne: "In Canada, first of all, there were the two races: each could be employed to balance the other" (p. 273). D'emblée, on remarque que ce jugement sur le Canada est plein de bonnes intentions en ce qu'il pose, sur le mode d'une complémentarité, la relation entre les cultures francophone et anglophone là où chacune pourrait, semble-t-il, équilibrer l'autre au nom de ce qui serait "un mieux vivre ensemble". Mais en quoi les travers de l'un peuvent-ils être surmontés grâce aux vertus de l'autre, et vice versa? Au nom de quelles finalités et totalités harmonieuses peut-on ainsi supposer cette fusion des caractères culturels? Au nom de quelle vision psychologisante de la culture (et de l'interculturel) peut-on ainsi spéculer sur quelque unité "complémentarisante", quelle soit canadienne ou non? Pour plaider un tel équilibre, ne faut-il pas déjà avoir une vision de ce qu'est l'équilibre ou l'harmonie et, en l'occurrence, celle du sujet canadien? Les deux cultures ont-elles seulement la même vision de cette supposée harmonie? Et quelle peut être la valeur de ce prétendu concept, l'équilibre (l'autre nom ici de l'harmonie), au nom

10. Cela dit, le discours critique au Canada anglais, sous l'influence notamment du postmodernisme, n'adhère pas à ce genre de discours identitaire tel que formulé par $\mathrm{H}$. MacLennan. On a désormais plutôt tendance à reconnaître l'hétérogénéité de l'identité canadienne (voir à ce sujet Frank Davey, PostNational Arguments. The Politics of the Anglophone-Canadian Novel since 1967, Toronto, University of Toronto Press, coll. "Theory/Culture", 1993). 
164

duquel on veut juger le devenir du sujet politique? N'anticipe-ton pas là le sujet de la communauté politique sur le seul mode d'une psychologie simpliste et non plus sur celui du politique et du droit?

Le développement de l'État moderne et de la politique internationale montre au contraire qu'il est possible, par-delà la finitude de telle ou telle inscription culturelle ou nationale, à la fois de s'entendre et de se (faire) reconnaitre comme sujet collectif; cette reconnaissance pouvant s'inscrire ensuite dans quelque texte constitutionnel. Cette scène (du) politique transcende les différences en même temps qu'elle les rassemble en les faisant (se) reconnaître. En considérant le Canada comme fondé sur un équilibre culturel d'ordre psychologique, on oublie que ce qui compte sur la scène politique, ce sont moins les bons sentiments - l'amitié ou la complémentarité - que l'esprit citoyen ou la civilité qui émane d'une conception du politique où prédomine la question de la reconnaissance.

Question de reconnaissance encore trop souvent refoulée par quelque déclaration d'amitié — sinon, d'amour! —, c'est-à-dire par la paradoxale violence des bons sentiments. 